Background and aims Aetiology of BPD is multifactorial with prenatal and postnatal factors being involved. First, we aimed to evaluate the association between chorioamnionitis and BPD. Secondly, the effect of other perinatal factors on the risk of developing BPD were analysed.

Methods Retrospective analysis of all infants with GA $<32$ weeks or BW $<1500$ g. admitted into our hospital between 2002-2010. 120 patients who died before 36 weeks of PMA were excluded.

Results The average GA was: $29,7 \pm 3$ s; $217 / 432$ (50\%) had any type of chorioamnionitis (histological or clínical); 75/432 (17.4\%) met diagnostic criteria for BPD at 36 weeks.

Univariate analysis: lower GA, any type of chorioamnionitis, DAP and duration of mechanical ventilation (MV) were associated with an increased risk of DBP $(\mathrm{p}<0.05)$.

Multivariate analysis: administration of antenatal steroids or chorioamnionitis did not independently modify the risk of BPD. But adding both, the effect became statistically significant protective for BPD (OR 0.52, 95\% CI 0.03 to 0.79 ).

Days in $\mathrm{MV}$ is the only factor that independently increased the risk of BPD. Neither a lower GA nor the presence of PDA had significantance; but, the risk of BPD was higher in the presence of PDA and MV together: every day in MV increased the risk of BPD (OR 1.130, 95\% CI1.001- 1.27).

Conclusions Chorioamnionitis in coexistence with antenatal corticosteroids decreases the risk of BPD. Mechanical ventilation is the main risk factor for BPD. In the presenceof DAP, ventilation increases the risk of BPD.

\section{PO-0758 LARYNGEAL MASK AIRWAY DEVICE PLACEMENT IN NEONATES}

${ }^{1} \mathrm{~A}$ Wanous, ${ }^{2} \mathrm{~A}$ Wey, ${ }^{2} \mathrm{~K}$ Rudser, ${ }^{3} \mathrm{~K}$ Roberts. ${ }^{1}$ Biological Sciences, University of Minnesota, Minneapolis, USA; ${ }^{2}$ Biostatistics, University of Minnesota, Minneapolis, USA; ${ }^{3}$ Neonatology, University of Minnesota, Minneapolis, USA

\subsection{6/archdischild-2014-307384.1397}

Background Endotracheal intubation (EI) is currently required for surfactant administration. However, EI is associated with adverse physiologic effects, including bradycardia and hypoxia. The laryngeal mask airway (LMA) may provide a more practical and less invasive alternative to EI for surfactant administration. Aim Determine feasibility of LMA placement in neonates by investigating the time, number of attempts and physiologic stability during placement of the device.

Methods Infants $\geq 1250$ g who required surfactant administration were eligible. Videotape of the LMA placement procedure was reviewed to determine number of attempts, duration of attempts, total procedure time, and heart rate and oxygen saturation change from baseline.

Results Twenty-two infants were included in analysis. Mean total procedure time was 129 seconds $( \pm 187)$. Duration of attempts was 59 seconds $( \pm 81)$. Successful placement was achieved on the first attempt in $73 \%$ of cases. Two attempts were required in $14 \%$ of cases and all procedures were successful in $\leq 3$ attempts. As compared to baseline, heart rate increased 3 beats per minute on average ( \pm 4 , range: -3 to 11$)$ and oxygen saturation decreased by $7 \%$ on average ( \pm 8 , range: -24 to 1$)$, as shown in Figure 1.

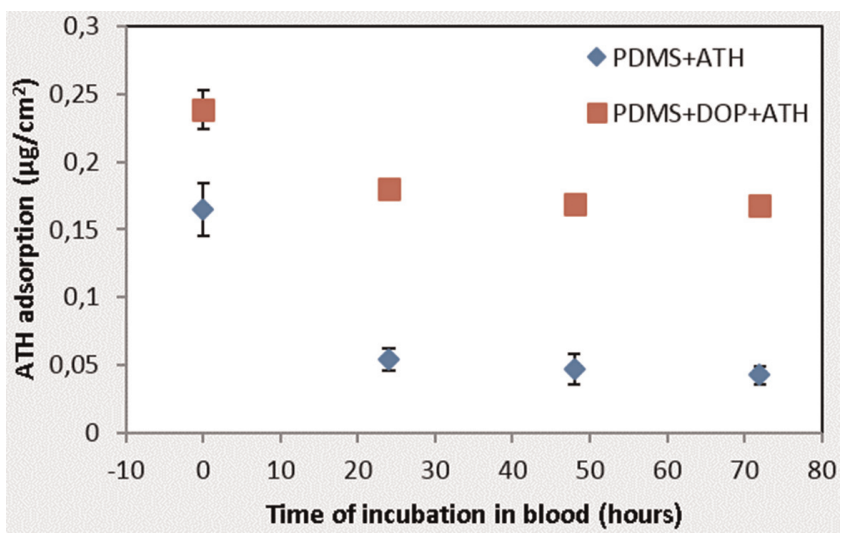

Abstract P0-0758 Figure 1

Conclusions Successful placement was achieved in the majority of patients in one attempt with an average total procedure time of approximately $2 \mathrm{~min}$. Physiologic parameters were maintained close to baseline with minimal fluctuation in heart rate and oxygen saturation. Placement of the LMA device is feasible in neonates.

\section{P0-0759 SURFACE MODIFICATION OF A POLYDIMETHYLSILOXANE MICROFLUIDIC OXYGENATOR WITH DOPAMINE AND A COVALENT ANTITHROMBIN-HEPARIN COMPLEX FOR THE PREVENTION OF THROMBOSIS}

${ }^{1} \mathrm{~J}$ Leung, ${ }^{2} \mathrm{~L}$ Berry, ${ }^{3} \mathrm{~N}$ Rochow, ${ }^{4} \mathrm{R}$ Selvaganapathy, ${ }^{2} \mathrm{~A}$ Chan, ${ }^{1} \mathrm{~J}$ Brash, ${ }^{3} \mathrm{C}$ Fusch. ${ }^{1}$ School of Biomedical Engineering, McMaster University, Hamilton, Canada; ${ }^{2}$ Thrombosis and Atherosclerosis Research Institute, McMaster University, Hamilton, Canada; ${ }^{3}$ Pediatrics, McMaster University, Hamilton, Canada; ${ }^{4}$ Mechanical Engineering, McMaster University, Hamilton, Canada

\subsection{6/archdischild-2014-307384.1398}

Introduction Prematurely born infants suffer respiratory insufficiency, our lab has developed a novel microfluidic oxygenator with polydimethylsiloxane (PDMS) gas transfer membranes to provide respiratory support. The objective of the work reported here was to modify the PDMS surfaces with a covalent antithrombin-heparin (ATH) complex to prevent coagulation

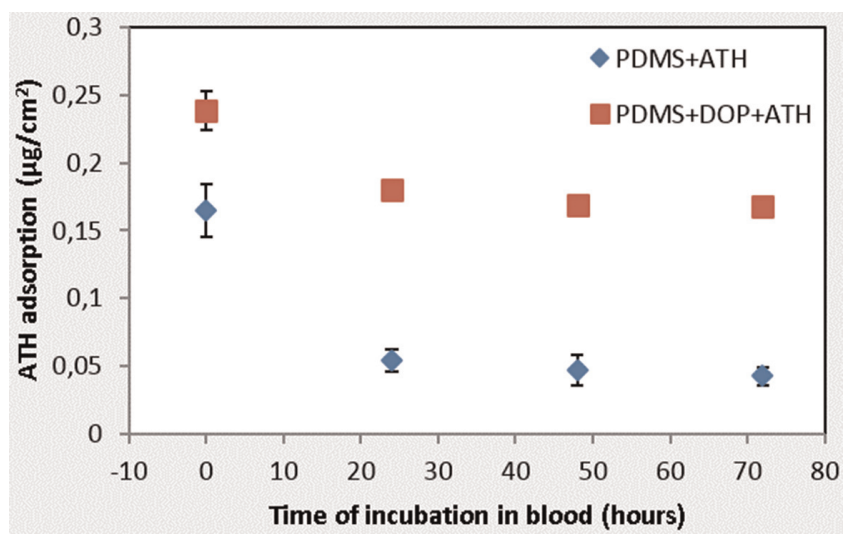

Abstract P0-0759 Figure 1 ATH desorption into blood from PDMS-ATH and PDMS-DOP-ATH surfaces 
and thrombosis due to blood-material contact. Specifically the ATH was attached to the PDMS using dopamine as a 'bio-glue'.

Materials and methods PDMS discs were incubated in a solution of dopamine hydrochloride and then in ATH solution. A separate set of PDMS discs were justed incubated in ATH. Uptake of ATH and adsorption of antithrombin (AT) from plasma (a measure of anticoagulant activity) to the various surfaces was measured using ${ }^{125}$ I-labelled ATH and AT. Stability of ATH on surfaces was evaluated by measuring residual radioactivity after incubation in blood.

Results ATH uptake on PDMS was higher with dopamine as glue (Fig. 1), $\sim 74 \%$ of the original ATH was lost from PDMS + ATH after $3 \mathrm{~h}$ in blood, whereas only $\sim 30 \%$ was lost from $\mathrm{PDMS}+\mathrm{DOP}+\mathrm{ATH}$.

The ATH surface with dopamine is adhesive, thus showed higher AT adsorption (42.3 ng/ $\mathrm{cm}^{2}$ ) compared to PDMS (6.3 ng/ $\mathrm{cm}^{2}$ ), and therefore should have higher anticoagulant activity.

Conclusions An antithrombin-heparin complex (ATH) was attached to PDMS using dopamine as a bio-glue. The use of dopamine gave surfaces with higher concentration and greater stability of ATH. The bound ATH showed potential for anticoagulant activity through extensive adsorption of antithrombin from plasma.

\section{P0-0760 RELIABILITY OF SINGLE-USE PEEP VALVES DURING MANUAL VENTILATION OF NEONATES}

JC Hartung, G Schmalisch, CC Roehr. Neonatology, Charité University Berlin, Berlin, Germany

\subsection{6/archdischild-2014-307384.1399}

Background and aim Current guidelines recommend selfinflating bags (SIB), flow-inflating bags and T-piece resuscitators for manual ventilation of neonates. They further recommend the use of PEEP. Using a SIB, PEEP can be provided by attaching a PEEP valve to the device. These valves are mostly reusable items. However, several studies could show that multi-use PEEP valves could only deliver insufficient levels of PEEPand that their reliability was further decreased by repeated sterilisation cycles.

The aim of our study was to test whether single-use PEEP valves reliably delivered the set PEEP.

Methods Ten new single-use PEEP valves from 5 different manufacturers (2 valves each from Laerdal $\left(5-20 \mathrm{cmH}_{2} \mathrm{O}\right), \mathrm{DROH}$ $\left(0-10 \quad \mathrm{cmH}_{2} \mathrm{O}\right)$, Vital Signs $\left(5-20 \mathrm{cmH}_{2} \mathrm{O}\right)$, medisize (5-20 $\left.\mathrm{cmH}_{2} \mathrm{O}\right)$, Ambu (0-20 $\left.\mathrm{cmH}_{2} \mathrm{O}\right)$ ) were attached to an electromechanically driven SIB to ventilate a manikin simulating a $1 \mathrm{~kg}$ preterm infant (PIP $20 \mathrm{~cm} \mathrm{H2O,} \mathrm{RR} \mathrm{60/min).} \mathrm{The} \mathrm{delivered}$ PEEP was measured and analysed.

Results The valves delivered a mean (SD) PEEP of 3.5 (1.9) $\mathrm{cmH}_{2} \mathrm{O}$ when set to $5 \mathrm{cmH}_{2} \mathrm{O}$ and 5.6 (2.9) $\mathrm{cmH}_{2} \mathrm{O}$ when set to $10 \mathrm{cmH}_{2} \mathrm{O}$. One valve could not deliver any PEEP, the second valve from the same manufacturer could only deliver $0.0(0.0)$ and $1.4(0.0) \quad \mathrm{cmH}_{2} \mathrm{O}$ when set to 5 and $10 \mathrm{cmH}_{2} \mathrm{O}$, respectively.

Conclusion Single-use PEEP valves could be used as an alternative to multi-use items to avoid damage caused by repeated sterilisation procedures. However, they could not reliably deliver the set PEEP. Operators should be aware of the valves ' poor reliability and test them before each use.

\section{P0-0761 CLINICAL AND EVOLUTIVE PECULIARITIES OF THE BRONCHOPULMONARY DYSPLASIA AND WILSON-MIKITY SYNDROME IN PREMATURE CHILDREN}

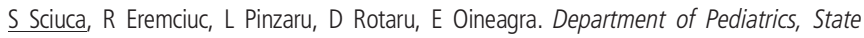
University of Medicine and Pharmacy, Chisinau, Moldova

\subsection{6/archdischild-2014-307384.1400}

Background Bronchopulmonary dysplasia (BPD) and WilsonMikity syndrom (WMS) are specific respiratory diseases in premature infants, and utilisation of advanced management techniques will increase the prognosis and life expectancy in children with BPD and WMS.

Aim To assess clinical features and impact of BPD and WMS on the appearance of chronic pulmonary diseases in premature children.

Methods The study pressents the results of a clinical and evolutive analysis of 10 children with BPD and 4 with WMS that were born premature with a brith weigth of 700-1400 g, gestational age $31.92 \pm 2,28$ weeks.

Results The comparative analysis showed clinical and explorative differences in children with BPD and those with WMS. Though the prematurity degree was similar, the onset of clinical signs in children with WMS was later comparing with those with BPD $(9,5 \pm 2,37$ vs $1,4 \pm 0.14$ days of life, $p<0.01)$. Respiratory symptoms in the first year of life were less persistent in children with WMS versus those with BPD, who still presented with suggestive imagistic sings (diffuse pulmonary nodular infiltrates accompanied by cystic changes and areas of hiperinflation). Pulmonary pathology progressed inchildren with BPD, causing death in 2 children at 3-5 months of life due to severe complication. In children with WMS, in evolution was favourable with fewer exacerbations, in 1 case with complete involution confirmed radiologically by the age of 1 year.

Conclusion BPD in premature children has high risks of progression into cronic pulmonary disease and death. In WMS the clinical signs appear later, are less severe and their evolution is more favourable.

\section{PO-0762 VOLUME TARGETED VENTILATION - EVIDENCE TO PRACTICE?}

${ }^{1} E$ Simons, ${ }^{2} R$ Dack, ${ }^{3} V$ Venugopalan. ${ }^{1}$ Paediatrics, Sandwell and West Birmingham NHS Trust, West Bromwich, UK; ${ }^{2}$ Paediatrics, Sandwell and West Birmingham NHS Trust, Birmingham, UK; ${ }^{3}$ Neonatal, Sandwell and West Birmingham NHS Trust, Birmingham, UK

\subsection{6/archdischild-2014-307384.1401}

Background and aim Volume targeted ventilation when compared to pressure controlled ventilation has been shown to reduce death and chronic lung disease in ventilated preterm neonates. ${ }^{1}$ Our audit assessed whether ventilated neonates born at Birmingham City Hospital, UK were appropriately converted to volume targeted ventilation as per the departmental guideline. Methods We collected retrospective data from all ventilated neonates born at Birmingham City Hospital, September 2012August 2013. We identified 125 neonates, but collected data from 76. 'Mechanical Ventilation in Neonates - Sandwell and West Birmingham Hospital NHS Trust Guideline', May 2012 was our standard and we aimed to achieve $100 \%$ compliance.

Results Of the 76 neonates, 35(46\%) were excluded due to being transferred in or out of the unit. Of the remaining 41 (54\%) neonates, 34\% were switched to volume targeted 